\title{
Présentation. Les commentaires dans l'Europe de la première modernité, entre interprétation et rhétorique
}

\section{Christine Noille}

\section{(2) OpenEdition Journals}

Édition électronique

URL : http://journals.openedition.org/rhetorique/866

DOI : $10.4000 /$ rhetorique.866

ISSN : 2270-6909

Éditeur

UGA Éditions/Université Grenoble Alpes

Édition imprimée

ISBN : 978-2-37747-177-5

Référence électronique

Christine Noille, «Présentation. Les commentaires dans l'Europe de la première modernité, entre interprétation et rhétorique », Exercices de rhétorique [En ligne], 13 | 2019, mis en ligne le 06 décembre 2019, consulté le 12 septembre 2020. URL : http://journals.openedition.org/rhetorique/866 ; DOI : https://doi.org/10.4000/rhetorique.866

Ce document a été généré automatiquement le 12 septembre 2020.

\section{c) (i) (2)}

Les contenus de la revue Exercices de rhétorique sont mis à disposition selon les termes de la Licence Creative Commons Attribution - Pas d'Utilisation Commerciale - Partage dans les Mêmes Conditions 4.0 International. 


\title{
Présentation. Les commentaires dans l'Europe de la première modernité, entre interprétation et rhétorique
}

\author{
Christine Noille
}

1 Sur des vers de Virgile... À la suite de Montaigne et de son chapitre III, 5 des Essais, c'est à un voyage que nous vous invitons, dans les traditions anciennes du commentaire de texte. Cette longue pratique, qui a laissé un corpus abondant, a transmis la mémoire des modèles antiques, dans la lettre de leurs textes comme dans leur esprit: elle a actualisé au fil des siècles leur lisibilité ; et elle a construit un monde avec leurs mondes. À l'occasion d'une journée d'études à l'origine des articles ici réunis ${ }^{1}$, le pari a été de confronter les analyses et les lectures en se focalisant sur un seul corpus, les commentaires consacrés à l'Énéide de Virgile, modèle à la fois herméneutique et discursif pour toute la culture européenne, de la Renaissance au XVIII siècle. Au gré de nos étapes, dans les pratiques herméneutiques des humanistes, dans les annotations des grammairiens, dans les montages éditoriaux des philologues et dans les exercices des rhétoriciens, nous avons alors poursuivi quelques interrogations résistantes, sur ce qu'est l'interprétation et sur les manières d'en sortir, sur les réseaux de sens qui font la forme d'un texte et au bout du compte, sur ce que lire veut dire - ou plutôt, sur ce que l' Énéide nous dit.

2 Car que signifie exactement le texte virgilien quand il énonce :

À ces mots, elle embrasse tendrement son époux, et le serre amoureusement entre ses beaux bras. Vulcain, jusqu'alors insensible, sent renaitre tout à coup sa première ardeur pour sa divine épouse. Un feu, qui ne lui est pas inconnu, pénètre dans ses veines, et se répand dans tous ses membres amollis. Ainsi l'éclair, qui s'échappe de la nue enflammée, vole en un instant d'un pôle à l'autre... En même temps il lui donne les plus vifs et les plus délicieux embrassements, puis il s'endort tranquillement sur son $\operatorname{sein}^{2}$. 
Plusieurs réponses nous sont parvenues, de versants éloignés de la pratique commentatrice. Toutes concourent cependant à reconnaître que ce texte signifie un peu plus qu'il ne dit. Ce qui donne, sous la plume de l'interprète Michel de Montaigne :

[La poésie] représente je ne sais quel air plus amoureux que l'amour même. Vénus n'est pas si belle toute nue, et vive, et haletante, comme elle est ici chez Virgile [...]. Ce que j'y trouve à considérer, c'est qu'il la peint un peu bien émue pour une Vénus maritale ${ }^{3}[\ldots]$.

Efficacité des mots tout autant que des choses, qu'un rhétoricien plus tardif confirmera ainsi à propos d'une séquence juste antérieure de la même scène ${ }^{4}$ :

Elle ne se sert d'aucun exorde, les caresses du lit conjugal ayant suffi à mettre son époux dans de bonnes dispositions ${ }^{5}$,

et qu'un Melanchthon aura résumé, un siècle et demi plus tôt, dans une formule suggestive : « exordium per insinuationem ${ }^{6}$ », Vénus ou la créature insinuatrice - « foemina ", la Femme par excellence, comme dira ailleurs Ferrazzi ${ }^{7}$ Alors, de Montaigne à Melanchthon et à Ferrazzi, de la Renaissance humaniste aux classes de rhétorique, de l'Europe méditerranéenne à l'Europe protestante, comment décrire ces commentaires, qui tous ont fait une place à la rhétorique du corps par-delà l'éloquence des mots? interprétation morale? ou description d'un dysfonctionnement rhétorique? Les deux sans doute, mais cela revient au même ici : sur les vers de Virgile, entre commentateurs des XVe-XVIII ${ }^{e}$ siècles, il a été possible de se mettre d'accord.

Or, cela ne sera pas toujours le cas. La problématique à l'initiative de ce dossier repose en effet sur les implicites du débat critique contemporain, qui opère deux gestes fondamentaux dans notre optique: il associe l'interprétation au commentaire et la rhétorique à l'analyse théorique; et il oppose systématiquement théorie et interprétation. C'est donc par l'histoire de ce clivage qu'il nous faut ici commencer, pour rappeler ensuite la fluidité et la perméabilité des pratiques telles qu'elles sont attestées par le corpus des annotations virgiliennes dans l'Europe de la première modernité, avant d'en revenir à une perspective plus théorique pour penser en dehors de l'antinomie théorie-interprétation une identité forte pour ces pratiques commentatrices qui sont effectivement informées par des disciplines hétérogènes, de la philosophie à la grammaire et à la rhétorique.

\section{Division}

5 Pour la critique contemporaine, il y a une différence radicale - épistémologique, méthodologique, éthique, idéologique - entre interpréter et analyser un texte, ou comme on dit par facilité, « décrire » quelque chose d'un texte ${ }^{8}$.

Différence épistémologique tout d'abord, entre deux finalités de savoir étrangères l'une à l'autre, une investigation philosophique sur la tension du sens littéral vers d'autres niveaux de sens d'une part; un savoir sur l'architexte d'autre part, autrement dit sur les procédures, les formes et les modes de fonctionnement des œuvres de langage. Au moment de se mettre au travail sur les textes, un choix stratégique préside bel et bien à la relecture, entre d'un côté s'engager dans la décision du sens et de l'autre s'atteler à la mise en formes de la textualité.

7 Différence et même opposition de méthode, ensuite, entre deux discours critiques, avec d'un côté ce que Genette nommait déjà dans Figures I un discours de reprise herméneutique du sens, en symbiose commentatrice (citationnelle) avec le fil du texte, 
et de l'autre côté un discours d'analyse et de mise à distance ${ }^{9}$. La séparation se fait ici sur les manières d'étudier les textes, entre deux positionnements de l'auctorialité critique, le pathos de l'herméneute d'une part, son engagement polémique dans la poursuite du sens; l'éthos du théoricien d'autre part, entre désengagement et domination.

Différence éthique, également, en l'occurrence une gestion pragmatique différente du débat intra-disciplinaire : pour les uns (les interprètes), la négociation polémique du dissensus dès lors que l'interprétation établit une tension herméneutique entre le texte et un au-delà doctrinal toujours sujet à contestation, qu'il s'agisse des intentions (interprétation intentionnaliste) ou des valeurs et des normes (interprétation finaliste) ; pour les autres (les analyticiens), la gestion conciliatrice du consensus dans une modélisation des formes dont le critère de pertinence est strictement technique, puisqu'interne au geste même de mise en forme. (On n'a jamais vu des rhétoriciens se battre pour savoir si le discours de Vénus à Vulcain commence avec ou sans exorde : car les deux descriptions sont très strictement correctes.) L'accord entre analyticiens repose de fait sur le seul consensus opérationnel (protocolaire) et permet de donner aux formes décrites, selon les époques, force de norme (le théoricien établissant en quelque sorte l'état de fait du texte) ou force de proposition (le théoricien inscrivant dans le texte la possibilité d'une forme).

Différence idéologique surtout, quant aux présupposés sur le statut et la définition du texte qui légitiment et motivent ces deux gestes critiques - une différence d'ordre culturel, qui porte sur les "pourquoi » et par là même sur les "comment", pour reprendre l'analyse de Michel Charles ${ }^{10}$, et qui engage deux conceptions pour le coup contradictoires concernant le rapport au texte: d'un côté, l'idée contemporaine de l'autorité et de la monumentalité du texte détermine une enquête qui en déploie toujours plus les richesses et la singularité; de l'autre, l'idée que le texte est le composé, la combinatoire - plus ou moins arbitraire, plus ou moins contingente -, de structures et de formes reproductibles et modifiables s'accorde avec une pratique manipulatrice et interventionniste, qui opère une sérialisation toujours possible $d u$ texte sur d'autres corpus exemplifiant des formes comparables, sur d'autres versions exemplifiant des formes modifiées.

Ou, si l'on veut, une culture du texte - que Michel Charles nomme «commentaire »contre une culture qu'il nomme "rhétorique » et dont la fonction est de "casser ${ }^{11}$ " l'autorité du texte, de le considérer comme un assemblage de possibilités, "un instrument à fabriquer d'autres discours ${ }^{12}$ ». - Et Michel Charles n'en finit pas alors, dans la culture à dominante commentatrice qui est la nôtre et qui permet à une société de s'identifier par un certain nombre de textes monumentalisés, de promouvoir une méthode d'analyse alternative qui fonctionne comme "moyen le plus efficace de mettre le texte à distance ${ }^{13}$ » et oppose au discours de la révérence et de la confortation un discours de la résistance et de la contestation.

11 Bilan de cet état de l'art pour notre $\mathrm{xxI}^{\mathrm{e}}$ siècle : il y a d'un côté l'interprétation et de l'autre la réflexion théorique, comme il y a d'un côté un engagement dans le sens et de l'autre une technique de mise en formes, d'un côté le commentaire et de l'autre l'analyse, d'un côté une investigation en profondeur dans le texte et de l'autre un positionnement en surplomb, d'un côté une lecture partisane et de l'autre une construction intellectuelle, d'un côté une sanctification et une sanctuarisation de la textualité et de l'autre un démontage jubilatoire. 

l'institution et dans les pratiques, on en conviendra bien volontiers. Mais elle tombe complètement à plat pour prendre en charge les pratiques commentatrices de la première modernité, comme en témoignent abondamment les annotations liminaires, marginales ou infra-paginales qui accompagnent et structurent les grandes éditions des textes antiques, au premier rang desquelles celles de l'Énéide.

\section{Circulation}

13 Tout va bien lorsque les objets, les méthodes et les finalités épistémologiques sont séparables et séparés et qu'il y a lieu de distinguer entre théorie et interprétation, entre analyse travaillant à la productivité des textes et commentaire travaillant à leur autorité.

14 Mais il arrive qu'il existe aujourd'hui des pratiques herméneutiques tournées vers la création et l'invention des possibles (confer les propositions de Sophie Rabau sur le travail philologique ${ }^{14}$ ), vers la variation et la défamiliarisation (confer les travaux d'Yves Citton sur l'actualisation lectoriale ${ }^{15}$ ), des pratiques herméneutiques qui intègrent ainsi une part de la contestation des textes et des méthodologies de possibilisation; et des pratiques rhétoriques défendant l'hypothèse méthodologique du commentaire au sein de notre culture du texte ${ }^{16}$; de même que sont attestées plus loin dans le temps, nous y revoilà, des pratiques commentatrices qui n'établissent pas de coupure entre travail herméneutique et travail rhétorique, paraphrase idéologique, explication grammaticale et description anatomique.

15 La leçon majeure qui ressort en effet des articles ici rassemblés est la fluidité des démarches et la circulation des traditions commentatrices : de Cristoforo Landino au $\mathrm{XV}^{\mathrm{e}}$ siècle à Charles Rollin, deux siècles et demi plus tard, les pratiques commentatrices ne sont jamais « pures », exclusivement grammaticales, philosophiques ou rhétoriques, parce que fondamentalement elles répondent à deux visées elles-mêmes composites, la visée pédagogique (dans notre livraison, Melanchthon, La Cerda, Ferrazzi, Rollin) et la visée éditoriale (Landino, Dolce, Marolles).

16 L'introduction du commentaire de texte dans les classes de grammaire et de rhétorique relève d'un enseignement par l'usus, par la lecture commentée ${ }^{17}$ : et fût-elle centrée sur l'une ou l'autre des deux disciplines, cette pratique de la lecture expliquée est solidaire d'un cursus studiorum qui se parachève dans la classe de philosophie, d'où un étoilement possible des remarques sur toute la gamme qui va de l'explication lexicale à la réflexion morale. Là est au demeurant la grande caractéristique des commentaires antiques de Virgile aux IV et $v^{e}$ siècles (ceux d'Aelius Donat, Servius, Macrobe, Tiberius Donat) : à la fois grammaticaux et rhétoriques (avec déclinaisons multiples: un Aelius Donat commentera d'abord en grammairien, par exemple), ils intègrent sans difficulté des annotations morales ou des interprétations allégoriques.

Si les savoirs des commentateurs pédagogues sont multiples, les scénographies éditoriales de la première modernité convoquent également des annotations abondantes, qui forment tout un appareil de couches métatextuelles, des titres aux manchettes et aux notes marginales, des préfaces aux résumés incipitiaux et aux commentaires intercalés - sans oublier les opérations typographiques à valeur sémantique, séquençage, intitulation, illustration, etc. Il peut arriver que l'édition 
distingue entre les remarques grammaticales, rhétoriques ou morales en les spécialisant dans une partie dédiée du dispositif opéral, mais la pratique est plus généralement à la fusion voire à la confusion, comme l'emblématise au $\mathrm{XVI}^{\mathrm{e}}$ siècle l'édition triplex du théâtre de Térence ${ }^{18}$. Dans le détail, les options disciplinaires restent cependant identifiables: les explications grammaticales (lexicales et syntaxiques) s'accompagnent souvent de référence à des usages comparables dans d'autres textes antiques; les remarques d'obédience rhétorique portent sur le genre, le plan et les ressources du discours virgilien quand il est argumentatif ; la transposition allégorique des énoncés littéraux ouvre à des enseignements moraux; l'identification des obscurités introduit des débats d'ordre philosophique ou théologique. Ajoutons qu'au demeurant la compatibilité de ces remarques entre elles n'est pas un fait récent : dans la tradition éditoriale des dialogues platoniciens, le dispositif est fixé depuis l'antiquité tardive qui, pour chaque dialogue, fait précéder les lemmes interprétatifs d'un préambule rhétorique chapeautant l'ensemble, où sont exposés les "principaux points » de l'argumentation, parmi lesquels son but, sa division et son mode ${ }^{19}$.

Qu'en est-il cependant du statut de toutes ces remarques métatextuelles? Relèventelles de l'annotation discontinue (de la description ponctuelle) ou forment-elles un commentaire, au sens où notre contemporanéité l'entend, un discours cohérent prenant en charge un texte en tant qu'il est un tout composé et non l'addition d'éléments autonomes? Et qu'en est-il subsidiairement de l'idéologie du texte qui les sous-tend ? Une différence s'établit ici entre les notes grammaticales et les autres ${ }^{20}:$ le commentaire lexical se caractérise précisément par son pointillisme et sa discontinuité : il ne prend pas en charge le sens global du texte mais en reste au sens des phrases et l'ensemble de ses énoncés ne produit pas littéralement un dispositif discursif continu, mais reste de fait une liste ouverte. Il n'en est pas de même pour les remarques rhétoriques, morales ou allégoriques, qui thématisent une totalisation littérale ou idéologique du texte, modélisant sa cohérence sémantique ou dépliant ses implications herméneutiques. L'éclatement en énoncés discontinus, au fil des items textuels annotés, ne doit pas faire illusion : ces commentaires peuvent se lire comme des résumés descriptifs à la suite (pour les descriptions rhétoriques : lesquelles peuvent même faire l'objet d'une publication séparée, sans le texte en regard ${ }^{21}$ ) ou comme une thèse en réseau (pour les interprétations idéologiques).

Dernier point à relever, qu'elles soient commentaires au sens plein ou annotations ponctuelles, et qu'elles soient analytiques ou interprétatives, il n'en reste pas moins que ces annotations virgiliennes participent toutes d'une idéologie du texte tournée vers l'idée d'une combinatoire et non vers la confortation d'une singularitée ${ }^{2}$. Les enjeux herméneutiques déploient le texte poétique sur des intertextes philosophiques, les notes grammaticales ouvrent l'énoncé sur une bibliothèque des usages, et l'anatomie rhétorique d'une cohérence morphologique est au service d'une sérialisation du texte sur d'autres corpus exemplifiant des formes comparables, sur d'autres versions exemplifiant des formes modifiées.

Il est enfin un dénominateur commun à toutes ces pratiques anciennes du commentaire de texte: leur proximité avec des opérations de récriture, qui contreviennent définitivement à un quelconque dogme de l'intangibilité du texte. Tout se passe en effet comme si le geste du commentaire était d'abord un geste interventionniste, suggérant du même coup la supériorité du commentateur sur le texte commenté ou pour le moins 
sa liberté - et l'inventivité en général de ces relectures dans ce qu'elles font au texte, tout autant (et parfois plus) que dans ce qu'elles en disent.

\section{Intégration}

21 Disons qu'ici, nous allons faire bouger les lignes : et par exemple, ne plus analyser les métatextes en termes de discours - les évaluant qui par rapport à leurs objets, leurs méthodes, leurs raisons... -, mais revenir en arrière, en amont, et les ressaisir comme la mise au propre d'un travail, l'écriture d'une relecture. Ce qui va nous intéresser, ce n'est donc pas le discours métatextuel, mais le dispositif métatextuel, autrement dit l'ensemble formé par le texte et le métatexte pour y voir à l'œuvre un énoncé composite unique, dont certains énoncés (les énoncés métatextuels) interviennent sur le texte, sur sa structuration, sur le rythme de son déploiement, sur sa cursivité, sans cependant en changer une seule lettre. Telle est la voie pour substituer aux typologies clivantes des discours métatextuels une typologie intégrative des dispositifs métatextuels selon les modalités de leur intervention sur le syntagme textuel.

Il s'agira ici d'étendre le geste d'intervention à toute pratique métatextuelle, de la plus citationnelle à la plus distancée: titres, manchettes, en-têtes, résumés, notes, commentaires interlinéaires, commentaires autonomisés, discours théoriques..., tous, dès lors qu'ils accompagnent la lecture d'un texte, exemplifient un dispositif d'intervention sur le matériau textuel tout autant qu'ils dénotent un certain nombre de propositions sur le texte. Tous font quelque chose au texte avant que de faire quelque chose de leur propre discours ${ }^{23}$. Une des formes du commentaire juxtalinéaire, l'annotation philologique, nous servira ici de paradigme sensible, de matérialisation visuelle sur ces dispositifs immatériels que sont les dispositifs métatextuels.

Lisons donc quelques vers de Virgile dans une des éditions de la première modernité : un appel de note nous propose de nous interrompre. En lecteurs idéaux, à la fois compétents et coopératifs, nous nous interrompons, nous nous déportons vers la lecture de la note, nous revenons au texte dont elle est l'annotation pour mieux en mesurer la portée, puis nous refermons cet espace de va-et-vient entre note et texte annoté pour reprendre notre lecture de la suite du texte. Rien de plus, mais rien de moins.

24 De fait, le dispositif relectorial est ici exhibé dans son intégralité : il délimite le point d'insertion et l'extension de sa référenciation, il désynchronise la lecture cursive, l'interrompant, la dupliquant, la reprenant; il multiplie les contextes pertinents, des cotextes amont et aval aux énoncés métatextuels. Dans le premier cas on parlera d'un dispositif de référenciation; dans le deuxième, d'un dispositif de scansion textuelle; dans le troisième, d'un dispositif de parallélisation cotextuelle.

Premier point, donc, en quoi le dispositif métatextuel de référenciation est-il une intervention sur le texte? Comme le montrent les différents types d'annotation et de citation (de la plus précise : citation in extenso; à des formes moins lourdes : appel de note et note infrapaginale; et jusqu'aux plus modalités les plus distancées : évocation d'un passage textuel par une mention, un titre, un mot), la référence à un syntagme textuel nous oblige à deux manipulations sur le tissu textuel: nous le coupons (à l'endroit du point d'insertion) et nous délimitons l'extension du point d'impact (le métatexte porte-t-il sur un mot? un paragraphe? un épisode ${ }^{24}$ ?). Autrement dit, toute référenciation, aussi distancée et non citationnelle soit-elle, nous amène à identifier un 
espace défini du tissu textuel, à introduire dans le tissu textuel un morcellement et une focalisation. Retenons-en qu'en tant qu'il est un dispositif référentiel, le métatexte introduit dans le syntagme du texte un séquençage; il opère ce faisant un aménagement de la cursivité textuelle; et la mise en forme du texte qu'il produit est une structuration par délimitation.

Deuxième point, en quoi le dispositif métatextuel de scansion textuelle est-il une intervention sur le matériau textuel? Très clairement, l'irruption d'un métatexte suspend (temporairement ou définitivement) la poursuite du texte et introduit dans le temps de ma lecture des lectures supplémentaires : qu'il s'agisse d'une relecture de la séquence commentée ou de la lecture de la séquence de commentaire. Le dispositif métatextuel resynchronise ainsi le texte en interpolant des interruptions et un certain nombre de lectures désynchronisées (lectures réitératives, lectures additionnelles), en le ponctuant donc par des effets de suspension, de reprise et d'allongement. Retenonsen qu'en tant qu'il est un dispositif de scansion du texte, le métatexte introduit dans la dynamique textuelle une ponctuation différente ; il opère ce faisant un aménagement de la temporalité lectoriale (de la durée et de la vitesse de la lecture); et la mise en forme du texte qu'il produit est une structuration par réordonnancement du rythme.

Troisième point, enfin, en quoi le dispositif métatextuel de parallélisation cotextuelle estil une intervention sur le texte? L'étoilement du texte sur des séquences métatextuelles n'arrête pas de le décontextualiser et de le recontextualiser, la chose est connue: non seulement la séquence textuelle est mise en réseau avec la séquence métatextuelle ; mais, plus décisif quant à la forme du texte commenté, le métatexte repense la coordination des énoncés textuels, en la défaisant ou au contraire en la justifiant. D'un côté, deux séquences textuelles qui ne sont pas à la suite l'une de l'autre peuvent en effet être mises en relation par les séquences métatextuelles qui les commentent. De l'autre côté, l'enchaînement de deux séquences textuelles se succédant peut être raisonné, parce que les séquences métatextuelles qui les commentent prennent en charge une réflexion sur leur place et leur ordre dans un ensemble plus global ${ }^{25}$. Dans les deux cas, nous retiendrons que le métatexte est un dispositif de parallélisation des séquences textuelles qui aboutit à un montage du texte (par redistribution et/ou coordination); il opère ce faisant un aménagement des cotextes ; et la mise en forme du texte qu'il produit est une structuration par mise en réseau.

L'on voit dès lors en quoi appréhender le métatexte comme dispositif relectorial revient à en souligner la force réorganisatrice : avant que de produire un discours plus ou moins autonomisable, plus ou moins subordonné, le dispositif métatextuel intervient massivement sur la textualité sans en transformer la lettre, grâce à tout un travail de mise en forme: soit qu'il délimite et focalise une séquence; soit qu'il resynchronise le rythme du texte selon des processus de suspension, de récurrence et de reprise; soit qu'il parallélise les séquences textuelles en les coordonnant ou les redistribuant. Ces interventions, nommons-les par leur vrai nom : ce sont bel et bien des opérations de recomposition du texte. Elles aménagent l'organisation du texte dans l'espace et dans le temps, elles le modélisent et le reconfigurent - sans en changer la moindre lettre. textualité. Telle est, tout aussi bien, la grande leçon des montages qu'opèrent les 
commentaires anciens, des plus interprétatifs aux plus rhétoriques: lecture néoplatonienne de l'Énéide, avec la grande œuvre de Landino ( $\mathrm{xv}^{\mathrm{e}}$ siècle) que retrace ici Hélène Casanova-Robin ; annotations rhétoriques à visée morale qu'analysent Séverine Clément-Tarantino pour Tiberius Donat ( $v^{\mathrm{e}}$ siècle ap. J.-C.) et Florence de Caigny pour Michel de Marolles (xvII ${ }^{\mathrm{e}}$ siècle); commentaire en forme de réfection dans la récriture de Lodovico Dolce en 1571 que présente Valeria Cimmieri; remarques stylistiques à arrière-plan doctrinal pour Marolles, mais aussi pour une génération de rhétoriciens de la seconde moitié $d u x{ }^{e}$ siècle et $d u$ début $d u x{ }^{e}{ }^{e}$ siècle que confronte Christiane Deloince-Louette sur la question du style homérique; analyses spécifiquement rhétoriques enfin, mais travaillées par des impensés herméneutiques, que l'on se place, avec Francis Goyet, sur le plan des intentions, ou avec Christine Noille sur celui des finalités.

\section{NOTES}

1. Journée d'études du 22 avril 2011 à l'université Paris 7 Denis Diderot, intitulée «Les commentaires de l'Énéide à la Renaissance et à l'âge classique: entre rhétorique et interprétation »; organisée par Christine Noille-Clauzade pour l'équipe RARE - Rhétorique de l'antiquité à la Révolution (UMR 5316 Litt\&Arts / CNRS-Université Grenoble Alpes) dans le cadre du projet HERMES (ANR, Paris 7, dir. F. Lavocat). La présente publication s'inscrit également à la suite d'une précédente livraison d'Exercices de rhétorique (2|2013 - Sur Virgile, URL : https:// journals.openedition.org/rhetorique/172).

2. Virgile, Énéide, VIII, 387-392 et 404-406; traduction par l'abbé Desfontaines (Pierre-François Guyot), dans Les Euvres de Virgile, à Amsterdam, par la Compagnie des Libraires, 1743. Nous modernisons systématiquement l'orthographe. C'est cette citation, non traduite, que Montaigne donne intégralement dans son essai (voir note suivante).

3. M. de Montaigne, Les Essais, éd. P. Villey (1924), Paris, PUF, 1978, 1. III, chap. 5 « Sur des vers de Virgile », p. 849.

4. Énéide, VIII, 372-374, op. cit. : «Couchée dans un lit d'or à côté de son époux, elle lui tient ce langage, qui réveille sa tendresse: 'Mon cher époux, dans le temps que les rois de la Grèce assiégeaient Ilion [...]'. »

5. [M. A. Ferrazzi] Exercitationes rhetoricae in praecipuas P. Virgilii Maronis orationes, quae in Aeneidum libris leguntur, Padoue, Presses du Séminaire, 1694, «Discours 56. Vénus à son époux Vulcain : demande d'armes pour Énée » (sur Énéide, VIII, 374-386). Notre traduction s'appuie sur le travail de l'équipe RARE Rhétorique de l'Antiquité à la Révolution (UMR 5316 Litt\&Arts - CNRS / UGA). Sur Marco Antonio Ferrazzi (1661-1748), voir dans cette même livraison l'article de Francis Goyet, «Mythologies de la préméditation et mythologies de l'improvisation: sur quelques commentaires rhétoriques de l'Énéide ».

6. «Exorde par insinuation»: dans Ph. Melanchthon, Virgilius cum Phil. Melanchthonis scholiis, Hagueneau, Secerius, 1530 ; réédité sous le titre Enarratio Aeneidos Virgilii dans les Opera de Melanchthon, vol. XIX, éd. K. G. Bretschneider et H. E. Bindseil, Brunswick, Schwetschke et fils (Corpus Reformatorum), 1853, p. 459.

7. M. A. Ferrazzi, op. cit., « Discours 4. Vénus à Jupiter : plaidoyer pour les Troyens » (sur Énéide, I, 229-253). 
8. Nous reprenons ici un développement initialement paru dans notre article «La forme du texte: rhétorique et/ou interprétation ", Fabula-LhT, $\mathrm{n}^{\circ} 14$, février 2015; URL : http:// www.fabula.org/lht/14/noille.html, §. « Le roman de la division (l'Arbre ou la Source) ».

9. Voir Gérard Genette, «Structuralisme et critique littéraire », Figures I (1966), Seuil, « Points », 1976, p. 159-161.

10. Voir M. Charles, L'Arbre et la Source, Paris, Seuil, 1984, p. 101 : « [Le critique] choisit à la fois une méthode et un discours, [...] il ne peut prendre l'une sans prendre l'autre, [...] prenant, donc, le discours avec la méthode, il prend, avec le discours, tout un héritage. »

11. Ibid., p. 52 : «La rhétorique [...] cesse de "faire parler" infiniment le texte, elle cesse de s'y référer, elle casse son autorité. »

12. Ibid., p. 12.

13. Ibid., p. 52.

14. Voir le numéro de Fabula LHT que Sophie Rabau a dirigé sur la "Poétique de la philologie » (Fabula LHT n ${ }^{\circ}$ 5, nov. 2008, URL : https://www.fabula.org/lht/5/); en particulier sa présentation («La philologie et le futur de la littérature», ibid., URL: http://www.fabula.org/lht/5/ presentation.html) et son article intitulé " Pour une poétique de l'interpolation » (ibid., URL : http://www.fabula.org/lht/5/rabau.html).

15. Voir Y.Citton, Lire, interpréter, actualiser. Pourquoi les études littéraires?, Paris, Éditions Amsterdam, 2007.

16. Au premier rang desquelles les travaux de Michel Charles, en particulier : Introduction à l'étude des textes, Paris, Seuil, 1995 ; et Composition, Paris, Seuil, 2018.

17. Voir Fr. Goyet, «Charles-Joseph Hubert et la lecture rhétorique », Rivista Italiana di Filosofia del Linguaggio, 2017, p. 52-63 ; DOI : 10.4396/RHETPHIL201706.

18. Terentius, in quem triplex edita est P. Antesignani Rapistagnensis commentatio, Lyon, Macé Bonhomme, 1560 ; édition qui compile tous les commentaires disponibles en les fondant en un seul commentaire continu. Voir Ch. Deloince-Louette et J.-Y. Vialleton, «Présentation : Térence commenté, une école de rhétorique et de dramaturgie ", Exercices de rhétorique, 10 | 2017 ; URL : http://journals.openedition.org/rhetorique/557.

19. A. Lernould, "Commentaire", dans C. Berner et D. Thouard dir., L'Interprétation. Un dictionnaire philosophique, Paris, Vrin, 2015, p. 82.

20. Sur la spécificité du rhetor, voir Fr. Goyet, Le Regard rhétorique, Paris, Garnier, 2017.

21. Il en va ainsi pour les Exercitationes rhetoricae de Ferrazzi sur l'Énéide (op. cit.).

22. Pour une reformulation théorique de ce fait, voir Ch. Noille, «La forme du texte : rhétorique et/ou interprétation », op. cit., § « Le commentaire rhétorique : existence, résistance ».

23. Pour une première version de ce développement, voir ibid., $\S$ «Ce que tout métatexte fait au texte $»$.

24. Il s'agit là d'un enjeu capital dans la compréhension des commentaires rhétoriques anciens, puisqu'ils ne font référence qu'au premier mot de la séquence qu'ils décrivent («exorde», « amplification », « colère »)...

25. Sur cet impensé critique de la cursivité, voir Ch. Noille, «Le pion de la Tour. Analyse rhétorique des brouillons perdus du Quichotte", Fabula-LhT, $\mathrm{n}^{\circ}$ 17, 2016; URL: http:// www.fabula.org/lht/17/noille.html. 


\section{AUTEUR}

\section{CHRISTINE NOILLE}

Sorbonne Université - CELLF (UMR 8599) 